\title{
Pathophysiology of Glaucomatous Optic Neuropathy: Role of Optic Nerve Head Vascular Insufficiency
}

\author{
Sohan Singh Hayreh \\ Department of Ophthalmology and Visual Sciences, College of Medicine, University of lowa, lowa City, lowa, USA
}

\section{INTRODUCTION}

In 1970, based on my experimental and clinical studies, I defined glaucoma as: "a disease wherein the normal balance between the intraocular pressure and the blood pressure in the choroidal vessels supplying the optic disc and retrolaminar part of the optic nerve is disturbed, resulting in vascular insufficiency in the optic disc and retrolaminar part of the optic nerve, and hence in visual field defects and pathological changes in the optic disc and optic nerve." ${ }^{1}$ Since the conventional wisdom at that time was that glaucoma was essentially mechanical in nature, caused solely by high intraocular pressure (IOP), this concept was received with marked skepticism. Since then, however, evidence that vascular insufficiency in the optic nerve head (ONH) plays an important role in pathogenesis of glaucomatous optic neuropathy has progressively accumulated; so that now its vasogenic origin is widely accepted. ${ }^{2}$

To understand the vasogenic mechanism of glaucomatous optic neuropathy, it is crucial to have a good understanding of the following:

1. Blood supply of the ONH.

2. The various factors that influence the $\mathrm{ONH}$ circulation.

3. Evaluation of the $\mathrm{ONH}$ circulation.

\section{BLOOD SUPPLY OF THE OPTIC NERVE HEAD}

The subject of the blood supply of the ONH, while crucial in understanding the pathogenesis of glaucomatous optic neuropathy, anterior ischemic optic neuropathy and other ischemic disorders of the $\mathrm{ONH}$, has been beset by controversy over the decades. One of the reasons for this is the habit of extrapolating the in vivo ONH blood supply and flow pattern from postmortem cast, histological and morphological studies. All these aspects of blood supply of the ONH are discussed at length elsewhere. ${ }^{3,4}$ Briefly, from both the anatomical (Fig. 1) 1,6 $^{5,6}$ and blood supply (Fig. 2) $3,4,7$ points of view, the ONH consists of four distinct regions. These regions, from anterior to posterior aspect, are as follows.

\section{The Surface Nerve Fiber Layer}

This is the most anterior layer of the ONH. It contains the compact optic nerve fibers as they converge on to this part from all over the retina and bend to run back (Figs 1A).

This part is essentially supplied by the retinal arterioles (Fig. 2A). In few cases, however, the temporal part may be supplied by the posterior ciliary artery circulation from the underlying prelaminar region. When a cilioretinal artery (rarely a tiny ciliopapillary artery) is present, that supplies the corresponding part of the surface nerve fiber layer.

\section{The Prelaminar Region}

This part consists of optic nerve fibers arranged in bundles, surrounded by glial tissue septa, which contain capillaries (Fig. 1B).

There is overwhelming evidence that this region is supplied mainly by centripetal branches from the peripapillary choroid (Fig. 2). ${ }^{3,4,7}$ The blood supply in the peripapillary choroid, as well as in the $\mathrm{ONH}$, is sectoral. The peripapillary choriocapillaris and central retinal artery play no role in its blood supply.

\section{Lamina Cribrosa Region}

This forms a band of dense, compact connective tissue extending transversely across the entire width of the ONH (Fig. 1A). The lamina cribrosa is anchored firmly at the periphery to the surrounding sclera, centrally to the connective tissue envelope of the central retinal vessels, and posteriorly to the septa of the retrolaminar optic nerve (Fig. 1C). It is laminar in nature, with collagen bundles alternating with glial tissue. The diameter of the openings for the nerve fiber bundles varies greatly in different parts. Quigley et $\mathrm{al}^{8}$ showed that there is less connective tissue and larger pores in the superior and inferior than nasal and temporal parts, and the least amount in the inferoperipheral region. 


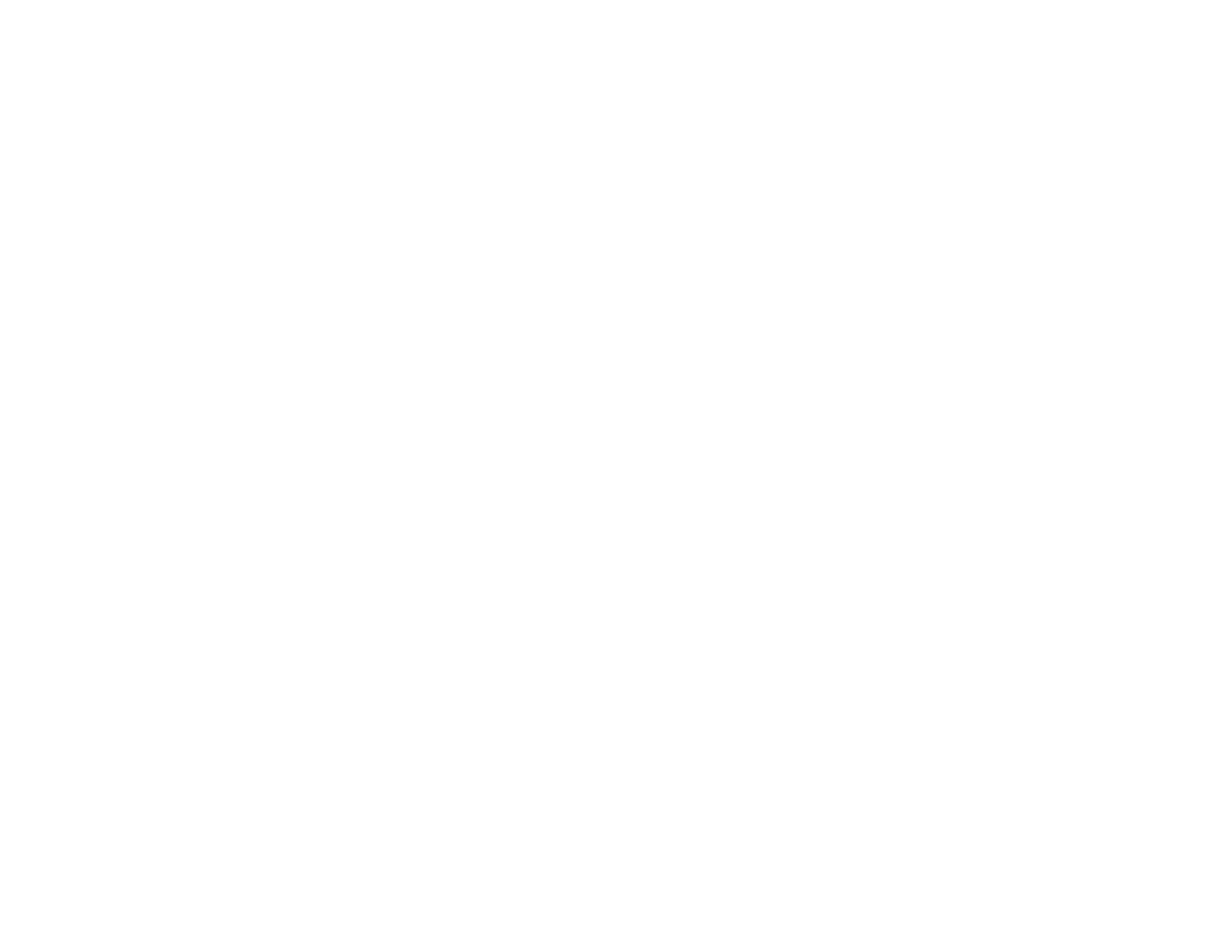

Figs 1A to D: Histological sections of the optic nerve head in rhesus monkeys. (A) Longitudinal section (LCR = Lamina cribrosa region; NFL $=$ Nerve fiber layer; PLR $=$ Prelaminar region; RLR $=$ Retrolamina region). (B-D) Transverse sections in prelaminar (B), lamina cribrosa, $(C)$ retrolaminar regions, and (D) note the presence of many vessels in the septa of the lamina cribrosa region

The lamina cribrosa region is supplied by centripetal branches arising directly from the short posterior ciliary arteries or from the intrascleral Circle of Zinn and Haller, when that is present (Fig. 2). In the lamina cribrosa, the blood vessels, 10 to $20 \mu$ in diameter, lie in the fibrous septa and form a dense capillary plexus (Figs 1C), which makes this part of the $\mathrm{ONH}$ a highly vascular structure. ${ }^{9}$ The central retinal artery does not supply this region.

A combination of the facts that: (a) the sizes of the pores in the lamina cribrosa and the thickness of the septa in-between the pores vary so widely in different part of the lamina cribrosa, and (b) the septa are essential vascular bundles, may play a role in differential susceptibility of different parts of the ONH to ischemia. Thinner septa would distort much more readily than the thicker septa; that in turn would result in more distortion of the blood vessels in thinner bundles and interference with the circulation.

\section{Retrolaminar Region}

This part of the optic nerve lies immediately behind the lamina cribrosa. It is enclosed by dura, arachnoid and pia (Fig. 1A). Here the nerve fiber bundles lie in polygonal spaces formed by the connective tissue septa (Fig. 1D). The septa are attached in the periphery to the pia, centrally to the connective tissue envelope around the central retinal vessels, and anteriorly anchored to the lamina cribrosa. The septa are essentially vascular bundles and form a complicated intercommunicating tubular framework for the nerve fiber bundles. The nerve fibers in this part are myelinated but are unmyelinated anterior to that.

This region has centripetal and centrifugal vascular systems (Fig. 2).

a. The centripetal system is the main and consistent vascular system, formed primarily by the recurrent pial branches from the peripapillary choroid and the Circle of Zinn and Haller (or the short posterior ciliary arteries), with additional pial branches from the central retinal artery and maybe from other orbital arteries. ${ }^{10-12}$ Branches from the pial vessels run centripetally in the septa.

b. A centrifugal system may be seen in some nerves and consists of a few inconstant branches from the central retinal artery.

In summary, the primary source of blood supply to the ONH is the posterior ciliary artery circulation via the peripapillary choroid and short posterior ciliary arteries (or the Circle of Zinn and Haller, when present) (Fig. 2). 

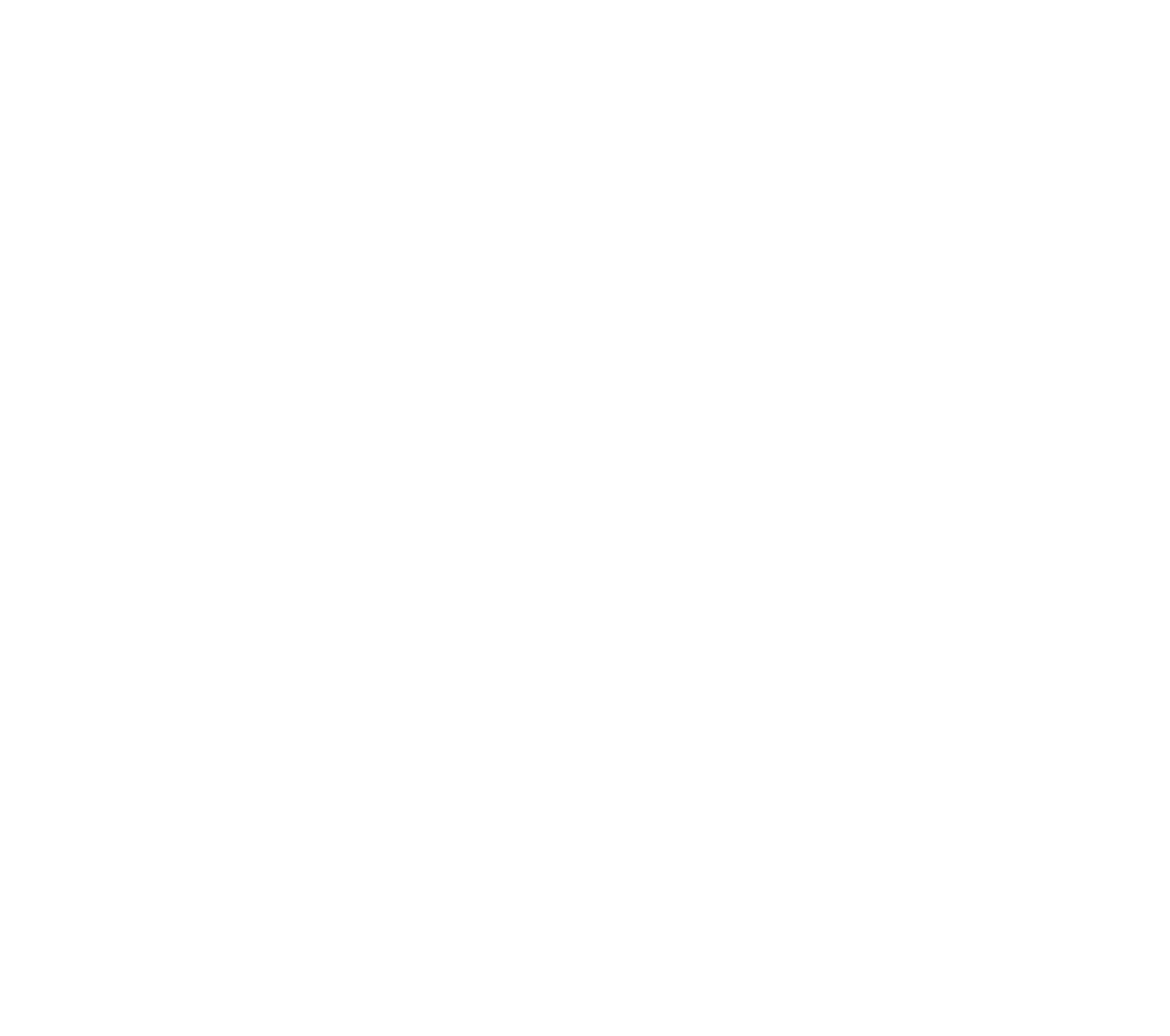

Figs 2A and B: Schematic representation of blood supply of: $(A)$ the optic nerve head and $(B)$ the optic nerve. A reproduced from Hayreh ${ }^{6}$ ) [B modified from Hayreh, SS (1974) Trans Am Acad Ophthalmol Otolaryngol; 78:OP240-OP254.]

Abbreviations used: $\mathrm{A}=$ arachnoid; $\mathrm{C}=$ choroid; Col. $\mathrm{Br} .=$ Collateral branches; $\mathrm{CRA}=$ central retinal artery; $\mathrm{CRV}=\mathrm{central}$ retinal vein; $\mathrm{CZ}=$ Circle of Zinn and Haller; $D=$ dura; $L C=$ lamina cribrosa; NFL = surface nerve fiber layer of the disc; OD = optic disc; ON = optic nerve; $P=$ pia; $\mathrm{PCA}=$ posterior ciliary artery; $\mathrm{PR}$ and $\mathrm{PLR}=$ prelaminar region; $\mathrm{R}=$ retina; $\mathrm{RA}=$ retinal arteriole; $\mathrm{S}=\mathrm{sclera} ; \mathrm{SAS}=$ subarachnoid space

\section{Interindividual Variations in the Blood Supply of the ONH}

When dealing with the role of blood supply of the $\mathrm{ONH}$ in various ischemic disorders of the $\mathrm{ONH}$, there is almost a universal misconception that all ischemic disorders can be explained based on one universal vascular pattern of the $\mathrm{ONH}$. This has resulted in confusion and controversy. It is crucial to realize that there is no one universal blood supply pattern of the ONH; there is a marked interindividual variation in it;; therefore there is marked variation in the pattern of ischemic disorders among various eyes. The subject of interindividual variation in the blood supply of the $\mathrm{ONH}$ is discussed at length elsewhere. . $^{3,4,13,14}$
Interindividual variations in the blood supply of the $\mathrm{ONH}$ are caused by infinite variation in the following.

i. The anatomical vascular pattern of the ONH: This varies widely.

ii. The number and pattern of distribution of the various posterior ciliary arteries: Usually there are 2 to 3 posterior ciliary arteries in an eye (Figs 3 to 6), called medial and lateral posterior ciliary arteries, but there may be 1 to 5 of them. The area supplied by each posterior ciliary artery varies widely from eye to eye (Figs 3 to 6 ). ${ }^{14}$

iii. The relationship of the watershed zone(s) between the posterior ciliary arteries to the ONH: Watershed zone is the border between the territories of distribution of 


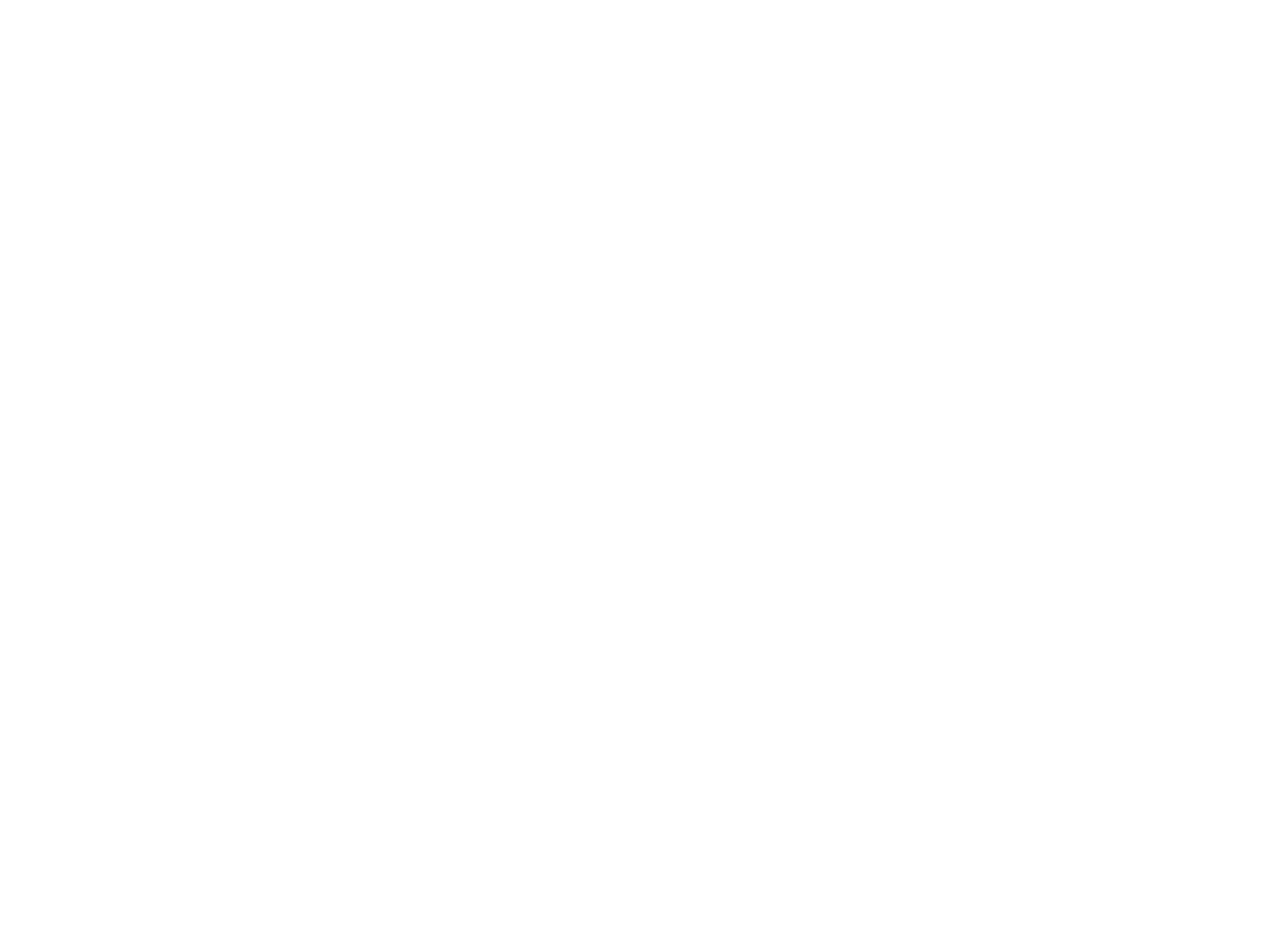

Figs 3A to D: Fluorescein fundus angiograms of four eyes with nonarteritic anterior ischemic optic neuropathy showing different locations of the watershed zone between the medial and lateral posterior ciliary arteries (vertical dark band and arrow) in relation to the optic disc. (A) Right eye with the watershed zone lying temporal to the optic disc. (B) Right eye with the watershed zone passing through the temporal part of the disc and adjacent temporal peripapillary choroid. (C) Left eye with the optic disc lying in the center of the watershed zone; (D) Left eye with the watershed zone passing through the nasal part of the disc and adjacent nasal peripapillary choroid. A reproduced from Hayreh ${ }^{2}$ and others from Hayreh ${ }^{13}$ )

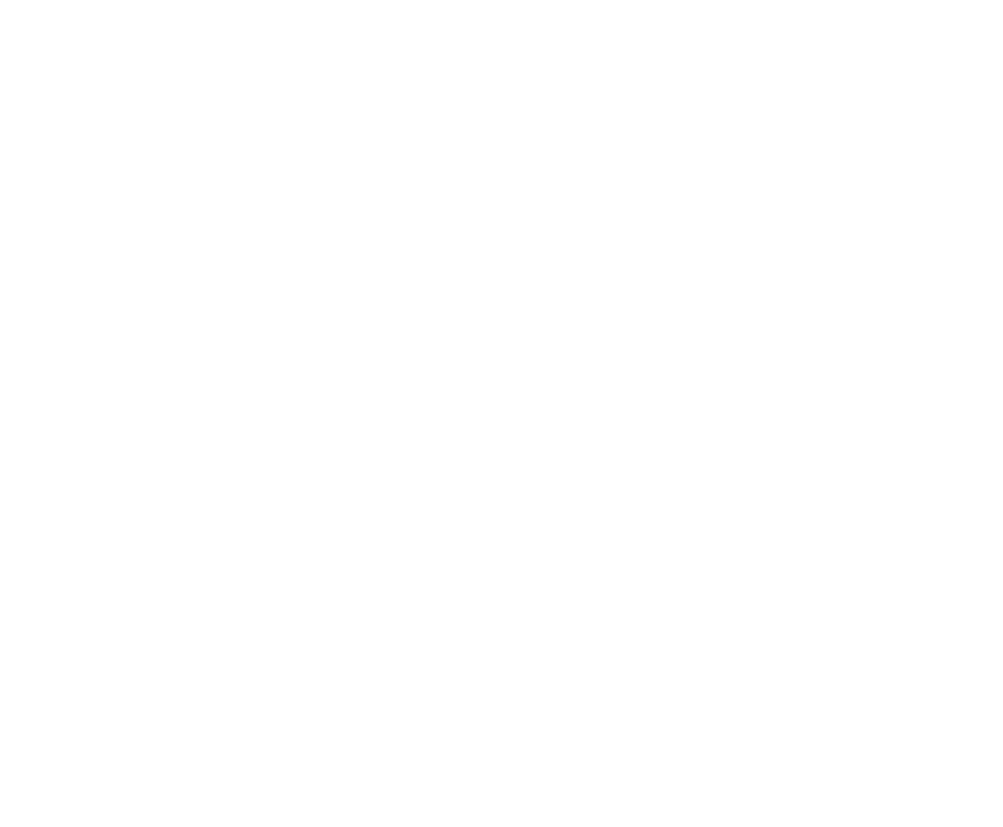

Fig. 4: Diagrammatic representation of some of the locations of the watershed zones (shaded area) between the medial and lateral posterior ciliary arteries in human eyes: (upper left) the shaded area represents the area within which the watershed zone may be situated. The remaining five diagrams are some examples of variations in the location. Fig. 3 shows fluorescein angiograms of some of these locations of the watershed zone (Reproduced from Hayreh ${ }^{14}$ ) 


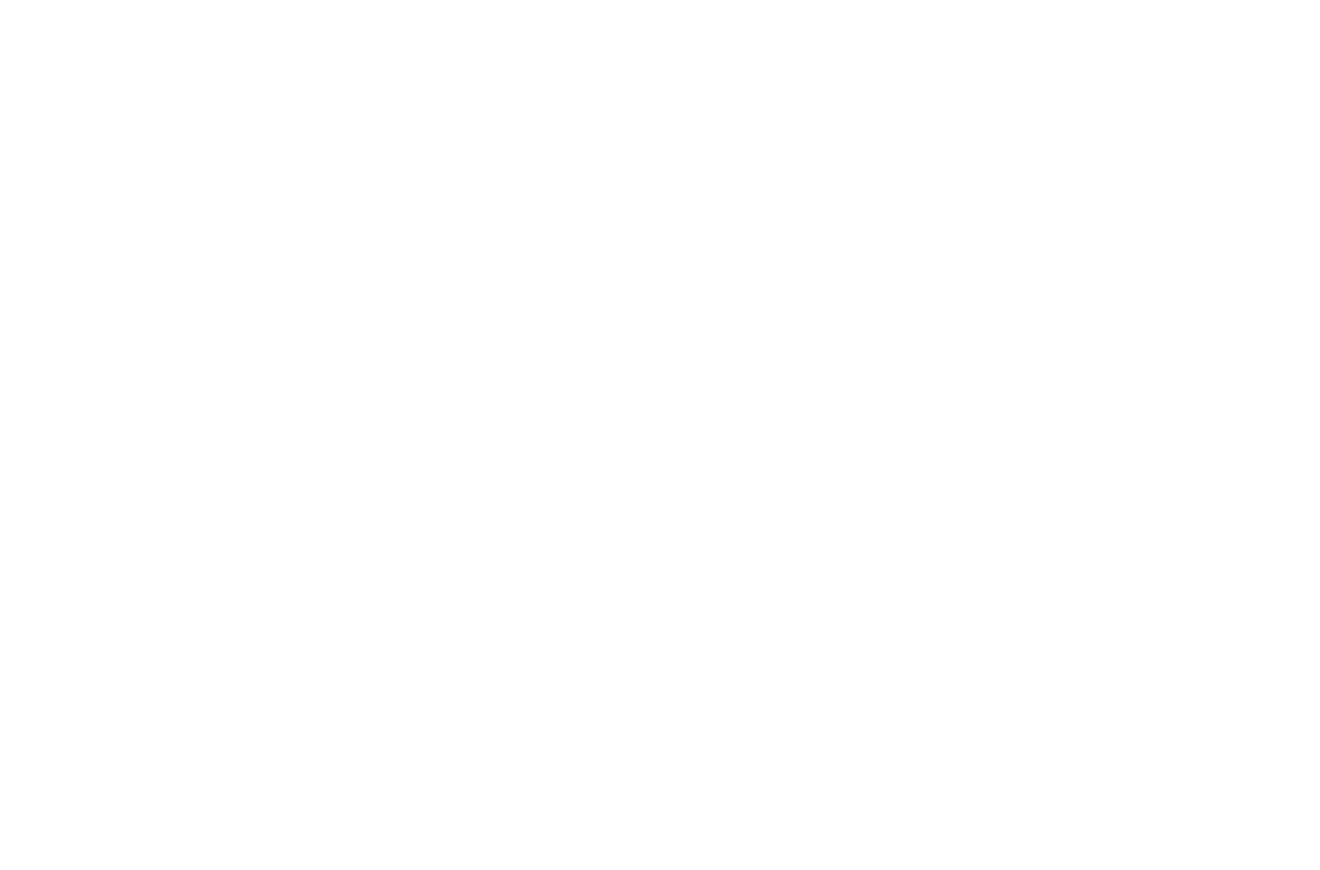

Figs 5A to F: Diagrammatic representations of some examples of the locations of the borders between the territories of the various posterior ciliary arteries: (A) with two posterior ciliary arteries: one medial and the other lateral; (B-D) with three posterior ciliary arteries in different combinations: $(B, C)$ have one medial and two lateral posterior ciliary arteries, and (D) one lateral and two medial; and (E) with four posterior ciliary arteries : two medial and two lateral. (F) Fluorescein fundus angiogram of right eye with normal tension glaucoma and cupping of the lower half of the optic disc with corresponding visual field defect. It shows non-filling of lower half of the watershed zone (indicated by arrow) and the involved lower half of the optic disc. In earlier phases of angiography, inferior temporal posterior ciliary artery showed markedly delayed filling (A-E reproduced from Hayreh ${ }^{14} ; \mathrm{F}$ from Hayreh ${ }^{13}$ )

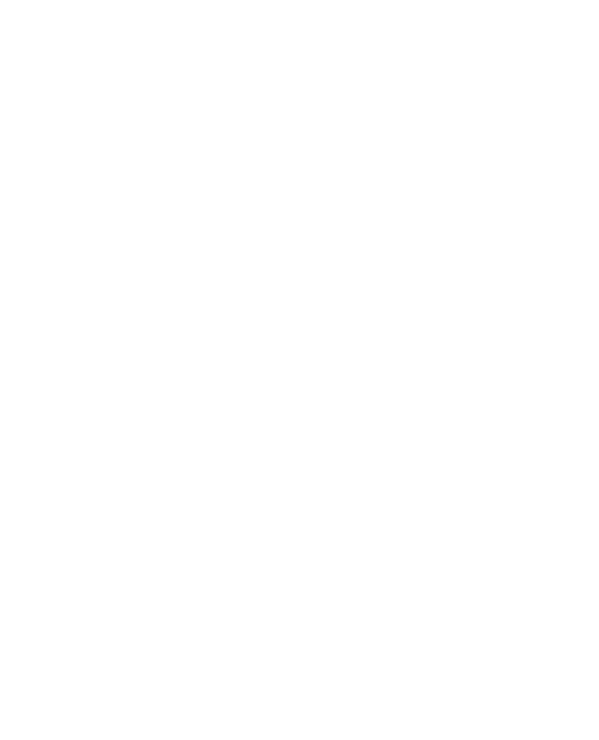

Fig. 6: Diagrammatic representation and incidence of some of the locations of the watershed zones (shaded area) between the medial and lateral posterior ciliary arteries seen in eyes with glaucomatous optic neuropathy. (Reproduced from Hayreh ${ }^{14}$ ) adjacent end-arteries. Posterior ciliary arteries are endarteries; therefore, there are watershed zones between the various posterior ciliary arteries. ${ }^{14}$ The significance of the watershed zone is that in the event of a fall in the perfusion pressure in the vascular bed of one or more of the endarteries, the watershed zone, being an area of comparatively poor vascularity, is most vulnerable to ischemia. As seen in Figures 3 to 6, ONH shows a marked interindividual variation relationship to the location of the watershed zone between the posterior ciliary arteries. This is an important factor in determining not only the vulnerability of $\mathrm{ONH}$ to ischemia but also the site and severity of ischemia in the $\mathrm{ONH}$.

\section{Venous Drainage of Optic Nerve Head}

The venous drainage from the $\mathrm{ONH}$ is by the central retinal vein (Fig. 2B). In the prelaminar region, it also has connections with the peripapillary choroidal veins. In the event of central retinal vein occlusion behind the lamina cribrosa, this communication assumes importance in developing retinociliary collaterals (erroneously called optociliary shunts). 


\section{Conclusions about Blood Supply of the ONH}

a. The main source of blood supply to the $\mathrm{ONH}$ is the posterior ciliary artery circulation except for the surface nerve fiber layer, which is supplied by the central retinal artery circulation. This dual supply has important implications when trying to evaluate the $\mathrm{ONH}$ circulation by various methods (see below).

b. The blood supply pattern in the ONH shows marked interindividual variations.

c. The blood supply in the $\mathrm{ONH}$ has a sectoral distribution, which is responsible for sectoral nerve fiber bundle defects in ONH ischemic disorders.

d. Location of the watershed zones between the various posterior ciliary arteries may play an important role in the development of ischemic disorders of the $\mathrm{ONH}$, as well as, in determining their site and severity.

\section{PATHOPHYSIOLOGY OF VARIOUS FACTORS THAT INFLUENCE THE ONH CIRCULATION}

To understand the role of vascular insufficiency in the ONH in the pathogenesis of various ischemic disorders of the $\mathrm{ONH}$, it is fundamental to understand the blood flow in the $\mathrm{ONH}$ in health and disease and the various factors that influence it. Lack of knowledge about these issues has caused most of the controversy about the pathogenesis and management of various ischemic disorders of the ONH. These factors are discussed at length elsewhere. ${ }^{15,16}$

To calculate the ONH blood flow, the following formula is used:

Blood Flow $=\frac{\text { Perfusion pressure }}{\text { Vascular Resistance }}$

Perfusion pressure $=$ Mean arterial blood pressure (BP) in the ONH vessels (not in the brachial artery) minus the IOP. Perfusion pressure is also equal to mean arterial pressure minus venous pressure in any vascular bed. Normally the pressure in the central retinal vein at the optic disc is slightly higher than the IOP, so that for all practical purposes, IOP is usually considered a good index of the ocular venous pressure.

Mean BP = Diastolic BP + 1/3 (systolic minus diastolic BP).

Therefore, the blood flow depends upon three parameters: (1) vascular resistance, (2) BP, and (3) IOP. The pathophysiology of these 3 factors, as well as of some others that influence the ONH blood flow, is discussed at length elsewhere, ${ }^{15,16}$ and the following is a brief account.

\section{Vascular Resistance}

This, according to Poiseuille's Law, is (a) inversely proportional to the fourth power of the radius of the vessel, (b) directly proportional to blood viscosity and (c) the length of the vessel. Therefore, vascular resistance in the ONH depends upon the state and caliber of the vessels feeding the ONH circulation and rheological properties of the blood. The latter are influenced by a large variety of hematologic disorders, particularly those causing increased blood viscosity.

The state and caliber of the vessels feeding the ONH may be altered by many factors, including the following.

\section{A. Autoregulation of Blood Flow}

The goal of autoregulation is to maintain a relatively constant blood flow, capillary pressure and nutrient supply in spite of changes in perfusion pressure. The evidence shows that blood flow in both the retina and $\mathrm{ONH}$ has autoregulation. ${ }^{16}$ Autoregulation of blood flow is due to alteration in resistance to blood flow. It is generally thought that the terminal arterioles regulate the resistance to flow, i.e. they dilate to increase the blood flow when the perfusion pressure falls, and constrict to reduce the blood flow in arterial hypertension. But since there is a limit to how far the terminal arterioles can constrict or dilate, the autoregulation operates only within a certain critical range of perfusion pressure (Fig. 7); it becomes ineffective and breaks down when the perfusion pressure goes below or above this critical range. Under those circumstances, the blood flow is directly proportional to the perfusion pressure. Thus, contrary to the general belief, the presence of autoregulation does not automatically regulate the blood flow at all levels of perfusion pressure (Fig. 7).

Breakdown of blood flow autoregulation in the ONH: Many systemic and local factors can cause this. The systemic causes include aging, arterial hypertension (Fig. 7), diabetes mellitus, marked arterial hypotension from any cause, arteriosclerosis, atherosclerosis, hypercholesterolemia, vasospasm and probably regional vascular endothelial disorders. ${ }^{17-20}$ It is also possible that many other, as yet unknown, causes can derange the autoregulation; possibly, also, some persons are born with

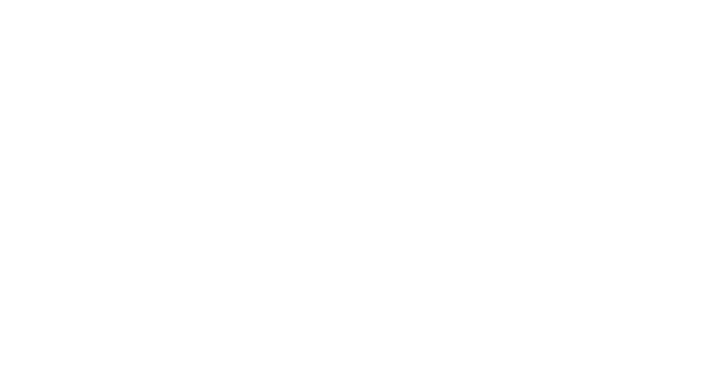

Fig. 7: A diagrammatic representation of range of blood flow autoregulation at different perfusion pressures in normal persons and in hypertensives (Hyperten). Green denotes range of presence of autoregulation and red its absence (Reproduced from Hayreh ${ }^{48}$ ) 
defective autoregulation, for instance, those who suffer from orthostatic arterial hypotension for no apparent reason.

\section{B. Vascular Endothelial Vasoactive Agents}

These agents are formed by vascular endothelium. Recent studies have shown that they play an important role in modulating the local vascular tone and most probably also in blood flow autoregulation, and they also regulate platelet function, coagulation and vascular growth. ${ }^{21}$ The vascular endothelial cells release various known endothelial vasoactive agents, which include prostanoids, nitric oxide, endothelins, angiotensins, oxygen free radicals, smooth muscle cell hyperpolarization, thromboxane $\mathrm{A}_{2}$ and other agents. ${ }^{21-24}$ Reninangiotensin system also exists in the vessel wall and plays a significant part in vasomotor control, ${ }^{25-29}$ apart from playing an important role in the control of arterial BP. Endothelium also plays a role in regulation of fibrinolysis because plasminogen activators and inhibitors are synthesized by the endothelium, ${ }^{30,31}$ and in thrombus formation by affecting the platelet aggregation, adhesion and other properties through prostacyclin, nitric oxide and other agents. ${ }^{21}$ Endothelial cells regulate vasomotor function not only by the various endothelial derived vasoactive agents but also they can function as mechanosensors and can transduce the mechanical signals produced by the physical force of blood flow into a biochemical signal to which the vessel can respond; ${ }^{32}$ this may be responsible for flow dependent changes in circulation.

Pathophysiological changes in the vascular endothelial cell structure and/or function occur in most major cardiovascular diseases, including atherosclerosis, diabetes mellitus, hypertension and ischemia. ${ }^{21,33-42}$

Vascular tone depends upon a balance between the endothelial vasodilators (e.g., nitric oxide) and vasoconstrictors (e.g., endothelin), so that reduced formation of vasodilators would result in vasoconstriction and vice versa (Fig. 8). Production of nitric oxide is impaired in several conditions, e.g., essential hypertension, ischemia and fall of arterial oxygen level. ${ }^{33,35,39,40-42}$ Therefore, endothelial cells play an important role in modulating the microvascular tone and blood flow autoregulation. As far the role of endothelial derived vasoactive agents in the $\mathrm{ONH}$ and ocular ischemia is concerned, a number of studies have reported the important role played by endothelin1 and nitric oxide in modulating the local vascular tone and regulating blood flow in the ophthalmic, posterior ciliary and retinal arteries and retinal and ONH vessels. ${ }^{15,16}$ Arteriosclerosis, hypercholesterolemia, aging, arterial hypertension, diabetes mellitus, ischemia and other causes so far unknown in the regional ocular and $\mathrm{ONH}$ vessels may be associated with abnormalities in production of endothelial derived vasoactive agents, thereby influencing the vascular resistance and blood flow in the $\mathrm{ONH} .{ }^{20}$ Experimental studies

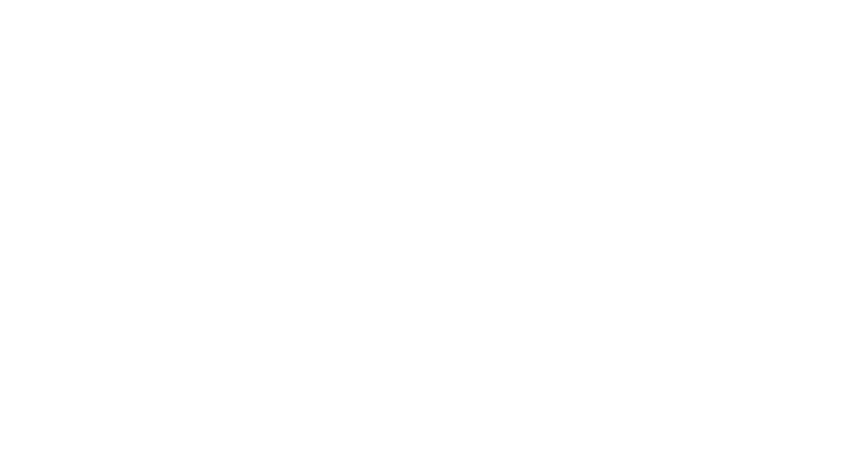

Fig. 8: A diagrammatic representation of the balance between nitric oxide (vasodilator) and endothelin-1 (vasoconstrictor) in modulating the local vascular tone

have shown that perineural chronic infusion of endothelin-1 on the anterior optic nerve in rabbits and rhesus monkeys produced a dose-dependent vasoconstriction, associated with a significant decrease in blood flow, increased optic disc cupping in rabbits, and diffuse loss of axons in monkeys. ${ }^{43-46}$ Thus, an understanding of the role of vascular endothelial vasoactive agents is crucial in any consideration of ONH circulation and ischemic disorders.

\section{Vascular Changes in the Arteries Feeding the ONH Circulation}

Arterial changes, which alter the vascular resistance in the internal carotid artery, ophthalmic artery and posterior ciliary arteries and/or smaller vessels in the ONH itself, may be produced by vasospasm, arteriosclerosis, atherosclerosis, vasculitis, drug-induced vasoconstriction or dilatation, and a host of other systemic and cardiovascular diseases.

\section{Arterial Blood Pressure}

According to the blood flow formula (see previous page), perfusion pressure in the $\mathrm{ONH}$ vascular bed determines the blood flow in the ONH. Perfusion pressure in the ONH vascular bed in turn depends mainly upon the arterial $\mathrm{BP}$ in the $\mathrm{ONH}$ vessels. Almost invariably, the BP is measured in the brachial artery during the waking hours; that, however, does not usually reflect the $\mathrm{BP}$ in the $\mathrm{ONH}$ vessels, for the following reasons:

i. There is a progressive fall of BP from the carotid artery to ophthalmic artery, posterior ciliary artery, onto the small vessels in the ONH. So that normally the actual BP in the $\mathrm{ONH}$ vessels may be half or even less than that measured in the brachial artery.

ii. If there are vascular changes in the internal carotid artery, ophthalmic artery and posterior ciliary arteries and/or smaller vessels in the ONH itself (produced by vasospasm, arteriosclerosis, atherosclerosis, vasculitis, drug-induced 
vasoconstriction, or other systemic and cardiovascular diseases), then the BP in the ONH capillaries would be even much lower than in normal individuals.

iii. During sleep, nocturnal arterial hypotension lowers the BP significantly from that recorded during waking hours (Fig. 9). ${ }^{47-52}$

Thus, it is important to realize that clinical BP measurement during waking hours in the brachial artery very much overestimates the actual BP in the ONH vessels. Since clinically measured $\mathrm{BP}$ is invariably regarded to represent $\mathrm{BP}$ in the $\mathrm{ONH}$, that erroneous impression has resulted in controversy of the role of $\mathrm{BP}$ in the pathogeneses of ischemic disorders of the $\mathrm{ONH}$.

Both arterial hypertension and hypotension can influence the ONH blood flow in a number of ways.

\section{A. Arterial Hypertension}

Both chronic arterial hypertension and malignant arterial hypertension can interfere with the ONH blood flow. This may be due to a number of mechanisms in these patients, including the following.

i. In arterial hypertension, there is increased vascular resistance in terminal arterioles all over the body-the basic pathology present in arterial hypertension.

ii. Arterial hypertension produces abnormalities in blood flow autoregulation. These may be produced by a variety of factors, including the following: (a) abnormalities in endothelial derived vasoactive agents [particularly reduction in production of nitric oxide (see previous parge)], (b) hypertension-induced adaptive change in blood flow autoregulation (Fig. 7), ${ }^{48,53}$ and (c) direct effect on the ONH blood flow by circulating vasoconstrictor agents (angiotensin and other circulating vasoconstrictor agents) in arterial hypertension. ${ }^{17,54}$

iii. Abnormal arterial hypotension produced by aggressive treatment of arterial hypertension. This has now emerged as a major problem with the aggressive use of highly potent hypotensive drugs currently available (see below). This may seem a paradoxical phenomenon-arterial hypertensives developing arterial hypotension, but this is a factor which is invariably overlooked when evaluating and managing $\mathrm{ONH}$ ischemic disorders. ${ }^{52}$

\section{B. Arterial Hypotension}

In an ONH with defective autoregulation, a fall of BP below a critical level must decrease its blood flow. A fall of BP in the $\mathrm{ONH}$ may be due to systemic or local hypotension.

i. Systemic arterial hypotension: The most common causes of systemic arterial hypotension are nocturnal arterial hypotension (Fig. 9) ${ }^{47,48,50,55}$ and intensive antihypertensive medication in hypertensives (with beta-

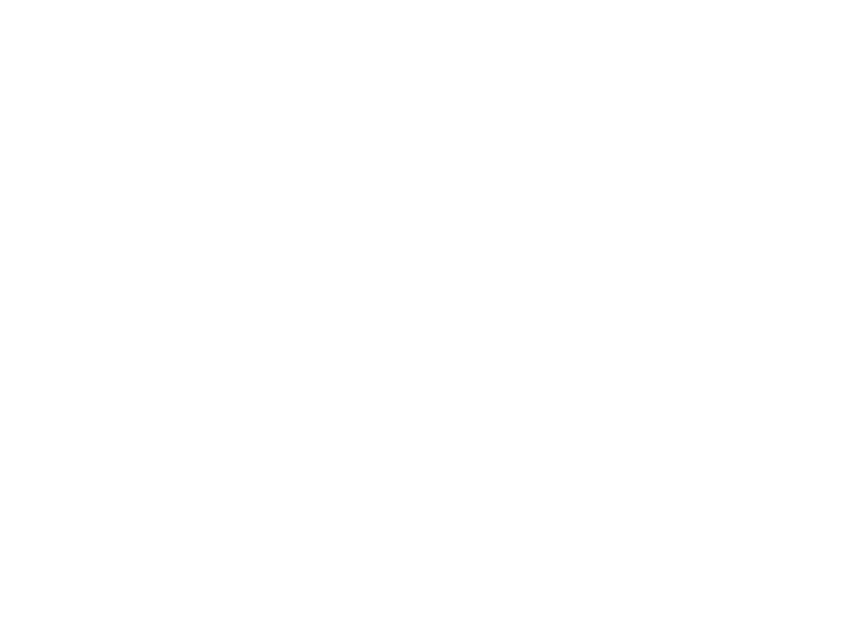

Fig. 9: Ambulatory blood pressure and heart rate monitoring record (based on individual readings), staring at $11 \mathrm{am}$ and going on until 10 am next day. The individual was not on any medication. Note that the $\mathrm{BP}$ is perfectly normal during the waking hours but shows a marked nocturnal arterial hypotension (with BP about $80 / 40 \mathrm{mmHg}$ ) during sleep. (Reproduced from Hayreh ${ }^{50}$ )

blockers, calcium-channel blockers and/or ACE inhibitors and other highly potent hypotensive drugs); ${ }^{48-51}$ less common causes include massive blood loss or shock. ${ }^{56}$ Recent studies have shown an association between nocturnal arterial hypotension and glaucomatous visual loss. ${ }^{48,50,55,57-61}$ Nocturnal arterial hypotension also plays a role in the development of non-arteritic anterior ischemic optic neuropathy, because at least $75 \%$ of these patients discover visual loss on waking in the morning. ${ }^{62}$

ii. Local arterial hypotension: This may be due to narrowing of the regional arteries, such as the internal carotid, ophthalmic or one or more of the posterior ciliary arteries, or arterioles supplying the $\mathrm{ONH}$, or a fall of perfusion pressure locally in the peripapillary choroid which supplies the ONH (Fig. 2A). As mentioned above, under such circumstances, the BP measured in the brachial artery grossly overestimate the $\mathrm{BP}$ in the $\mathrm{ONH}$ vessels.

\section{Intraocular Pressure}

As mentioned above, the perfusion pressure is equal to mean BP minus IOP. Thus, there is an inverse relationship between IOP and perfusion pressure in the $\mathrm{ONH}$, if the autoregulation is defective. In persons with normal $\mathrm{BP}$ and autoregulation, a much greater rise in IOP would be required before the $\mathrm{ONH}$ blood flow is compromised (e.g. in ocular hypertensives). By contrast, in persons with arterial hypotension, defective autoregulation or other vascular risk factors, even "normal" IOP may interfere with the ONH blood flow (e.g., in normal tension glaucoma). That may be of importance in the 
pathogenesis of glaucomatous optic neuropathy, particularly normal tension glaucoma. $2,47,50$

Assuming a supine position causes a short-term rise in IOP. As a part of circadian variation, the IOP is higher during sleep than during waking hours and is highest early in the morning. ${ }^{63-67}$ The recurrent spikes of raised IOP above normal levels during sleep (due to supine position and also sleep) may be completely missed during routine IOP measurement in clinic visits. However, these spikes in IOP, combined with the development of concurrent nocturnal arterial hypotension during sleep (Fig. 9), may constitute an important hidden risk factor for ONH ischemia in vulnerable ONHs. When dealing with the pathogenesis of glaucomatous optic neuropathy, this hidden risk factor is not always considered.

It has been claimed or implied in some studies that certain topically applied ocular hypotensive drugs (e.g., selective or nonselective beta-blockers, alpha agonists, carbonic anhydrase inhibitors, etc.) influence the ONH blood flow by direct ocular penetration and effect on the ONH blood vessels. However, drugs instilled in the conjunctival sac cannot readily reach the $\mathrm{ONH}$ in adequate concentration by direct penetration; hence, there is no scientific basis for the assumption that they have a direct action on the ONH circulation. Some of the drug may be absorbed into the systemic circulation and indirectly influence the ONH circulation; for example, it has been shown that persons using topical beta-blocker eye drops developed significant nocturnal arterial hypotension and nighttime bradycardia than those not using them. ${ }^{68}$

\section{Conclusions about Various Factors that Influence the ONH Circulation}

The following crucial factors need to be considered when considering the pathogenesis of ischemic disorders of the $\mathrm{ONH}$ (including glaucomatous optic neuropathy) and their management.

i.. Blood flow autoregulation plays a crucial role in the $\mathrm{ONH}$ circulation and many factors derange it. An $\mathrm{ONH}$ with deranged autoregulation is highly vulnerable to ischemic disorders.

ii. Vascular endothelial vasoactive agents play an important role in modulating the microvascular tone and blood flow in the $\mathrm{ONH}$ circulation. Production of these agents is impaired in many systemic and local conditions, and that would secondarily interfere with the ONH circulation.

iii. Nocturnal arterial hypotension is an important risk factor for the development of ONH ischemic disorders. The daytime-recorded BP gives no information whatever about the BP during sleep (Fig. 9).

iv. A rise of IOP during sleep and concurrent development of nocturnal arterial hypotension may together constitute an important hidden risk factor for $\mathrm{ONH}$ ischemia in vulnerable ONHs.

\section{EVALUATION OF THE ONH CIRCULATION}

Many methods have recently been put forward to evaluate ONH circulation and enthusiastic claims made. This is because of the increased recognition of the role of vascular insufficiency in the $\mathrm{ONH}$ in glaucomatous optic neuropathy and non-arteritic anterior ischemic optic neuropathy - two common visual disabling diseases. I have discussed the subject at length elsewhere. ${ }^{69}$ A critical review of the various advocated methods, including color Doppler imaging, transcranial Doppler, laser Doppler flowmetry, scanning laser Doppler flowmetry, magnetic resonance imaging, pulsatile ocular blood flow method, fluorescein fundus angiography, scanning laser fluorescein angiography, and temperature measurement, revealed that all these methods have limitations, and do not always give scientifically valid information on the actual ONH blood flow. ${ }^{69}$ Most of the recently advocated methods employ laser technology, e.g. laser Doppler flowmetry, scanning laser Doppler flowmetry and the laser speckle method. All of them suffer from the following fundamental flaw which invalidates their claims about the ONH blood flow: the laser beam in all of them is focused on the surface of the optic disc to measure the amount of blood flow in the ONH. As discussed above, the surface layer of the optic disc is supplied by the retinal circulation (Fig. 2 ), whereas glaucomatous optic neuropathy and non-arteritic anterior ischemic optic neuropathy are due to vascular insufficiency in the deeper ONH circulation, supplied by the posterior ciliary artery circulation (Fig. 2). Proponents of these laser methods have claimed that laser beam penetrates into the deeper tissues and measures the blood flow in the deeper layers of the $\mathrm{ONH}$, but a recent controlled experimental study in monkeys showed that laser Doppler flowmetry predominantly measures the blood flow in the superficial layers of the $\mathrm{ONH}$ supplied by the retinal circulation (Fig. 2). ${ }^{70}$ The same applies to Heidelberg retinal flowmetry, ${ }^{71}$ and to the laser speckle method. Repeated claims of color Doppler imaging and pulsatile ocular blood flow methods measuring $\mathrm{ONH}$ blood flow are similarly invalid, for several reasons discussed in detail elsewhere. $^{69}$

The other common flaw has been to equate blood velocity with the amount of blood flow in the ONH; blood velocity does not provide information about the quantity of blood flow unless we know the size of the lumen of the vessels. That information is not provided by any of these methods. Therefore, none of the methods used so far provide scientifically valid information about the ONH blood flow and circulation, and one has to take the enthusiastic claims made by all these methods with marked skepticism.

\section{Conclusions about Evaluation of the ONH Circulation}

We do not have a single clinical method that gives scientifically valid information on the in vivo blood flow in the $\mathrm{ONH}$ - neither in health nor in disease. 


\section{OVERALL CONCLUSIONS}

This brief review of the blood supply of the ONH and the factors that influence the blood flow in the ONH gives some idea of the great complexity of the subject. In ischemic disorders of the $\mathrm{ONH}$, a large number of systemic and local factors acting in different combinations and to different extents may derange the circulation in the ONH. These risk factors may be:

1. Predisposing risk factors which make the ONH susceptible to ischemia, and

2. Precipitating risk factors which act as the final insult. ${ }^{2,47,49,72}$

Available evidence strongly suggests that $\mathrm{ONH}$ ischemic disorders are multifactorial in nature..$^{2,47,49,72,73}$ In such a multifactorial scenario, one set of risk factors may be responsible for ONH ischemia in one case and a very different set in another, and a particular risk factor may be present or critical in one case and not in another. Thus, each patient with $\mathrm{ONH}$ ischemic disorder may have a unique combination of systemic and local factors, which collectively produce $\mathrm{ONH}$ ischemic damage; no stereotyping is possible. Unfortunately, the widespread lack of understanding of the great complexity of ONH blood flow in health and disease, along with our inability to evaluate in vivo the ONH blood flow reliably, has caused much controversy on the subject of the role of vascular insufficiency in the ONH in glaucomatous optic neuropathy and non-arteritic anterior ischemic optic neuropathy.

\section{ACKNOWLEDGEMENTS}

I am grateful to my wife Shelagh for her help in the preparation of this manuscript, and to my secretary Ms. Georgiane Perret for her help with the references.

\section{REFERENCES}

1. Hayreh SS, Revie IHS, Edwards J. Vasogenic origin of visual field defects and optic nerve changes in glaucoma. $\mathrm{Br} \mathrm{J}$ Ophthalmol 1970;54:461-72.

2. Hayreh SS. Progress in the understanding of the vascular etiology of glaucoma. Curr Opin Ophthalmol 1994;5:26-35.

3. Hayreh SS. The optic nerve head circulation in health and disease. Exp Eye Res 1995;61:259-72.

4. Hayreh SS. The blood supply of the optic nerve head and the evaluation of it-Myth and reality. Prog Retin Eye Res 2001;20:563-93.

5. Hayreh SS. Optic disc changes in glaucoma. Br J Ophthalmol 1972;56:175-85.

6. Hayreh SS. Structure and blood supply of the optic nerve. In Heilmann K, Richardson KT (Eds): Glaucoma: Conceptions of a Disease. Stuttgart: Thieme; 1978;78-96.

7. Hayreh S. Blood supply of the optic nerve head and its role in optic atrophy, glaucoma and oedema of the optic disc. Br J Ophthalmol 1969;53:721-48.
8. Quigley HA, Addicks EM. Regional differences in the structure of the lamina cribrosa and their relation to glaucomatous nerve damage. Arch Ophthalmol 1981;99:137-43.

9. Levitzky M, Henkind P. Angioarchitecture of the optic nerve. II. Lamina cribrosa. Am J Ophthalmol 1969;68:986-96.

10. Hayreh SS. A study of the central artery of the retina in human beings. Thesis for the Master of Surgery, Panjab University, India, 1958.

11. Singh S (Hayreh SS), Dass R. The central artery of the retina II. Distribution and anastomoses. Br J Ophthalmol 1960;44:28099.

12. Hayreh SS. The central artery of the retina-its role in the blood supply of the optic nerve. Br J Ophthalmol 1963;47:651-63.

13. Hayreh SS. Inter-individual variation in blood supply of the optic nerve head. Its importance in various ischemic disorders of the optic nerve head, and glaucoma, low-tension glaucoma and allied disorders. Doc Ophthalmol 1985;59:217-46.

14. Hayreh SS. In vivo choroidal circulation and its watershed zones. Eye 1990;4:273-89.

15. Hayreh SS. Factors influencing blood flow in the optic nerve head. J Glaucoma 1997;6:412-425,1998;7:71.

16. Hayreh SS. Blood flow in the optic nerve head and factors that may influence it. Prog Retin Eye Res 2001;20:595-24.

17. Hayreh SS, Servais GE, Virdi PS. Fundus lesions in malignant hypertension. V Hypertensive optic neuropathy. Ophthalmology 1986;93;74-87.

18. Hayreh SS, Bill A, Sperber GO. Metabolic effects of high intraocular pressure in old arteriosclerotic monkeys. Invest Ophthalmol Vis Sci 1991;32:810.

19. Hayreh SS, Bill A, Sperber GO. Effects of high intraocular pressure on the glucose metabolism in the retina and optic nerve in old atherosclerotic monkeys. Graefes Arch Clin Exp Ophthalmol 1994;232:745-52.

20. Haefliger IO, Meyer P, Flammer J, Lüscher TF. The vascular endothelium as a regulator of the ocular circulation: A new concept in ophthalmology? Surv Ophthalmol 1994;39:123-32.

21. Davies MG, Hagen PO. The vascular endothelium. A new horizon. Ann Surg 1993; 218:593-609.

22. Vanhoutte PM. The endothelium: modulator of vascular smooth muscle tone. N Engl J Med 1988;319:512-3.

23. Rubanyi GM. Cardiovascular significance of endothelium-derived vasoactive factors (1st edn). Mount Kisco, NY: Futura; 1991.

24. Ryan US, Rubanyi GM. Endothelial regulation of vascular tone. (1st edn). New York: Marcel Dekker; 1992.

25. Dzau VJ. Vascular renin-angiotensin: a possible autocrine or paracrine system in control of vascular function. J Cardiovasc Pharmacol 1984;6(suppl 2):S377-S382.

26. Suzuki M, Ohyama T, Satoh S. Conversion of angiotensin I to angiotensin II and inactivation of bradykinin in canine peripheral vascular beds. J Cardiovasc Pharmacol 1984;8:244-50.

27. Dzau VJ. Significance of the vascular renin-angiotensin pathway. Hypertension 1986;8:553-59.

28. Dzau VJ. Implication of local angiotensin production in cardiovascular physiology and pharmacology. Am J Cardiol 1987;59:59-65. 
29. Lindsey CJ, Paiva AC, Bendhack LM. Effects of teprotide, captopril and enalaprilat on arterial wall kinase and angiotensin converting activity. J Hypertens 1987;5(suppl):S71-S76.

30. Todd AS. The histological localization of fibrinolysis activator. J Pathol Bact 1959;78:281-83.

31. Dosne AM, Dupuy E, Bodevin E. Production of a fibrinolytic inhibitor by cultured endothelial cells derived from human umbilical vein. Throm Res 1978;12:377-87.

32. Davies PF, Tripathi SC. Mechanical stress mechanisms and the cell: an endothelial paradigm. Circ Res 1993;72:239-45.

33. Ku DD. Coronary vascular reactivity after acute myocardial infarction. Science 1982;218:576-8.

34. Lüscher TF, Raij L, Vanhoutte PM. Endothelium-dependent responses in normotensive and hypertensive Dahl rats. Hypertension 1987; 9:157-63.

35. VanBenthuysen KM, McMurtry IF, Horwitz LD. Reperfusion after coronary occlusion in dogs impairs endothelium-dependent relaxation to acetylcholine and augments contractile reactivity in vitro. J Clin Invest 1987;79:265-74.

36. De Tejada IS, Goldstein I, Azadzoi K, Krane RJ, Cohen RA. Impaired neurogenic and endothelium-mediated relaxation of penile smooth muscle from diabetic men with impotence. $\mathrm{N}$ Engl J Med 1989;320:1025-30.

37. Tesfamariam B, Jakubowski JA, Cohen RA. Contraction of diabetic rabbit aorta due to endothelium-derived $\mathrm{PGH}_{2}-\mathrm{TXA}_{2}$. Am J Physiol 1989; 257:H1327-H1333.

38. Lüscher TF. Imbalance of endothelium-derived relaxing and contracting factors: a new concept in hypertension? Am J Hypertension 1990;3:317-30.

39. Pearson PJ, Schaff HV, Vanhoutte PM. Long-term impairment of endothelium-dependent relaxations to aggregating platelets after reperfusion injury in canine coronary arteries. Circulation 1990;81:1921-27.

40. Meyer PR, Muller JM, Tanner MA. Effects of oxygen tension on endothelium dependent responses in canine coronary microvessels. Cardiovasc Res 1991;25:885-94.

41. Clavier N, Kirsch JR, Hurn PD, Traystman RJ. Effect of postischemic hypoperfusion on vasodilatory mechanisms in cats. Am J Physiol 1994;267:H2012-H2018.

42. Forte P, Copland M, Smith LM, Milne E, Sutherland J, Benjamin N. Basal nitric oxide synthesis in essential hypertension. Lancet 1997;349:837-42.

43. Cioffi GA, Orgül S, Onda E, Bacon DR, Van Buskirk EM. An in vivo model of chronic optic nerve ischemia: the dose-dependent effect of endothelin-1 on the optic nerve microvasculature. Curr Eye Res 1995;14:1147-53.

44. Cioffi GA, Orgül S. The effects of chronic optic nerve ischemia in the rabbit. In Drance SM (Ed): Vascular risk factors and neuroprotection in glaucoma - Update. Amsterdam: Kugler Pub; 1996;115-22.

45. Orgül S, Cioffi GA, Bacon DR, Van Buskirk EM. An endothelin1 induced model of chronic optic nerve ischemia in rhesus monkeys. J Glaucoma 1996;5:135-8.

46. Cioffi GA, Sullivan P. The effect of chronic ischemia on the primate optic nerve. Eur J Ophthalmol 1999;9:S34-S36.
47. Hayreh SS, Zimmerman MB, Podhajsky P, Alward WLM Nocturnal arterial hypotension and its role in optic nerve and ocular ischemic disorders. Am J Ophthalmol 1994;117:603-24.

48. Hayreh SS. Duke-Elder Lecture: Systemic arterial blood pressure and the eye. Eye 1996;10:5-28.

49. Hayreh SS. Acute ischemic disorders of the optic nerve Pathogenesis, clinical manifestations, and management. Ophthalmol Clin North Am 1996;9:407-42.

50. Hayreh SS, Podhajsky PA, Zimmerman B. Role of nocturnal arterial hypotension in optic nerve head ischemic disorders. Ophthalmologica 1999;213:76-96.

51. Hayreh SS. The role of age and cardiovascular disease in glaucomatous optic neuropathy. Surv Ophthalmol 1999;43:S27S42.

52. Hayreh SS. Role of nocturnal arterial hypotension in the development of ocular manifestations of systemic arterial hypertension. Curr Opin Ophthalmol 1999;10:474-82.

53. Hayreh SS. Malignant arterial hypertension. Ophthalmol Clin North Am 1992;5:445-73.

54. Hayreh SS, Servais GE, Virdi PS. Fundus lesions in malignant hypertension. VI. Hypertensive choroidopathy. Ophthalmology1986;93:1383-1400.

55. Hayreh SS. Vascular factors in the pathogenesis of glaucomatous optic neuropathy. In Drance SM (Ed): International Symposium on Glaucoma, Ocular Blood Flow, and Drug Treatment. 1990, Seville, Spain. Baltimore: Williams \& Wilkins; 1992;33-41.

56. Hayreh SS. Anterior ischemic optic neuropathy. VIII. Clinical features and pathogenesis of post-hemorrhagic amaurosis. Ophthalmology 1987;94:1488-1502.

57. Béchetoille A, Bresson-Dumont H. Diurnal and nocturnal blood pressure drops in patients with focal ischemic glaucoma. Graefes Arch Clin Exp Ophthalmol 1994;232:675-9.

58. Graham SL, Drance SM, Wijsman K, Douglas GR, Mikelberg FS. Ambulatory blood pressure monitoring in glaucoma. Ophthalmology 1995;102:61-9.

59. Bresson-Dumont H, Béchetoille A. Rôle de la tension artérielle dans l'évolutivité des lésions glaucomateuses. J Fr Ophtalmol 1996;19:435-42.

60. Meyer JH, Brandi-Dohrn J, Funk J. Twenty four hour blood pressure monitoring in normal tension glaucoma. Br J Ophthalmol 1996;80:864-7.

61. Detry M, Boschi A, Ellinghaus G, De Plaen JF. Simultaneous 24-hour monitoring of intraocular pressure and arterial blood pressure in patients with progressive and no-progressive primary open-angle glaucoma. Eur J Ophthalmol 1996;6:273-8.

62. Hayreh SS, Podhajsky PA, Zimmerman B. Non-arteritic anterior ischemic optic neuropathy-Time of onset of visual loss. Am J Ophthalmol 1997;124:641-7.

63. Frampton P, Da Rin D, Brown B. Diurnal variation of intraocular pressure and the overriding effects of sleep. Am J Optom Physiol Opt 1987;64:54-61.

64. Brown B, Morris P, Muller G, Brady A, Swann PG. Fluctuations of intraocular pressure with sleep. I. Time course of IOP increase after the onset of sleep. Ophthalmic Physiol Opt 1988;8:24648. 
65. Wildsoe C, Eyeson-Annan M, Brown B, Swann PG, Fletcher T. Investigation of parameters influencing intraocular pressure increase during sleep. Ophthalmic Physiol Opt 1993;13:35765.

66. Buguet A, Py P, Romanet JP. 24-hour (nyctohemeral) and sleeprelated variations of intraocular pressure in healthy white individuals. Am J Ophthalmol 1994;117:342-47.

67. Zeimer RC. Circadian variations in intraocular pressure. In Ritch R, Shields MB, T Krupin T (Eds): The glaucomas (2nd edn). St. Louis: Mosby; 1996;429-45.

68. Hayreh SS, Podhajsky PA, Zimmerman B. Beta-Blocker eye drops and nocturnal arterial hypotension. Am J Ophthalmol 1999;128:301-9.

69. Hayreh SS. Evaluation of optic nerve head circulation: Review of the methods used. J Glaucoma 1997;6:319-30.

70. Petrig BL, Riva CE, Hayreh SS. Laser Doppler flowmetry and optic nerve head blood flow. Am J Ophthalmol 1999;127:41325.

71. Wang L, Cull G, Cioffi GA. Depth of penetration of scanning laser Doppler flowmetry in the primate optic nerve. Arch Ophthalmol 2001;119:1810-14.
72. Hayreh SS, Joos KM, Podhajsky PA, Long CR. Systemic diseases associated with non-arteritic anterior ischemic optic neuropathy. Am J Ophthalmol 1994;118:766-80.

73. Anderson DR. Glaucoma: the damage caused by pressure. Am J Ophthalmol 1989;108:485-95.

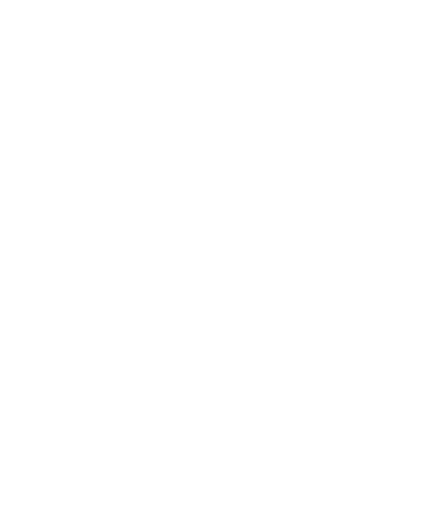

Dr Sohan Singh Hayreh (sohan-hayreh@uiowa.edu)

The mass of men worry themselves into nameless graves, while here and there a great unselfish soul forgets himself into immortality. 